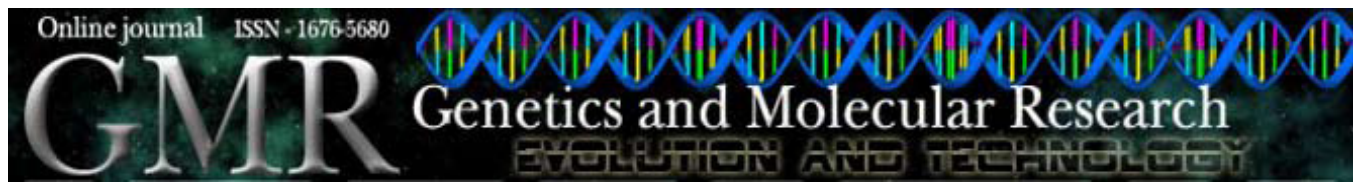

\title{
RNA-binding motif protein RBM22 is required for normal development of zebrafish embryos
}

\author{
F. He ${ }^{1,2 *}$, C.-T. Wang ${ }^{2 *}$ and L.-T. Gou ${ }^{2}$ \\ ${ }^{1}$ College of Life Science, Sichuan University, Chengdu, China \\ ${ }^{2}$ State Key Laboratory of Biotherapy and Cancer Center, West China Hospital, \\ West China Medical School, Sichuan University, Chengdu, China \\ *These authors contributed equally to this study. \\ Corresponding author: L.-T. Gou \\ E-mail: goulantu@gmail.com
}

Genet. Mol. Res. 8 (4): 1466-1473 (2009)

Received September 4, 2009

Accepted September 28, 2009

Published December 8, 2009

\begin{abstract}
RBM22 is a newly discovered RNA-binding motif protein, belonging to the SLT11 family; it has been reported to be involved in pre-splicesome assembly and to interact with the $\mathrm{Ca}^{2+}$-signaling protein ALG-2. However, previous studies have not demonstrated whether its expression is essential for early embryogenesis in vertebrates. We utilized zebrafish as a developmental model to study the role of RBM22 during embryogenesis. The aligned protein sequences of RBM22 were found to be highly conserved. In situ analysis showed that zRBM22 was expressed as early as the one-cell stage, implying maternal origin during oogenesis; embryos continued to express zRBM22 through at least the $32-\mathrm{h}$ postfertilization (hpf) stage of development. RT-PCR analysis confirmed the in situ expression pattern, indicating that RBM22 is expressed without any spatial and temporal specificity during zebrafish development. zRBM22 morpholino-induced alterations in development were observed as early as $15 \mathrm{hpf}$ of embryogenesis, based on a morphological analysis. Embryogenesis was monitored through $32 \mathrm{hpf}$; knockdown resulted in a truncated axis and developmental arrest of the head and tail. We conclude that RBM22 plays an important role in zebrafish embryogenesis.
\end{abstract}

Key words: RNA-binding motif protein; RBM22; Morpholino; Zebrafish; Embryo development 


\section{INTRODUCTION}

The spliceosome is a large RNA-protein complex, where pre-mRNA splicing proceeds to form the mature mRNA. Five small nuclear ribonucleoprotein particles (snRNPs) $\mathrm{U} 1, \mathrm{U} 2, \mathrm{U} 4, \mathrm{U} 5$, and $\mathrm{U} 6$ and a large number of protein splicing factors construct the major spliceosome (Kramer, 1996). Spliceosome assembly follows a carefully orchestrated stepwise pathway (Will and Luhrmann, 2001). It in turn generates the E (early) complex, the pre-spliceosomal A complex and the catalytic C complex (Nilsen, 2000).

Over 300 putative spliceosomal protein components have now been identified by proteomic approaches (Jurica and Moore, 2003); however, many unexpected proteins present, having no apparent direct connection to splicing, still await functional characterization. Some of the additional proteins identified in these complexes have known associations with other aspects of RNA processing, including transcription and mRNA export.

RBM22 is a new RNA-binding motif protein with a conserved RNA-binding domain, a zinc finger of the unusual type $\mathrm{C}-\mathrm{x} 8-\mathrm{C}-\mathrm{x} 5-\mathrm{C}-\mathrm{x} 3-\mathrm{H}$, and a $\mathrm{C}$-terminus that is unusually rich in the amino acids Gly and Pro, including sequences of tetraprolines (Figure 1, Figure 2A). RBM22 belongs to the SLT11 gene family. Slt11p, a splicing factor in yeast, is required for spliceosome assembly. It was identified on the basis of synthetic lethality with a mutation at the 5'end of U2 snRNA where it is involved in intermolecular U2/U6 helix II interaction. The RNA-annealing and -binding activities of Slt11p are two distinct biochemical properties (Xu et al., 1998; Xu and Friesen, 2001). As the homologue of SLT11 in vertebrate, recent studies indicated that RBM22 was involved in pre-splicesome assembly, functioned in alternative splicing, and was essential for vertebrate development (Chen et al., 2007; Barboric et al., 2009).

To further investigate the role of RBM22 in biological functions, we utilized zebrafish as a developmental model. Using a number of approaches available with zebrafish (Nasevicius and Ekker, 2000), we present data indicating that RBM22 has a functional role during zebrafish embryogenesis.

\section{MATERIAL AND METHODS}

\section{Fish and embryo maintenance}

Zebrafish (Danio rerio), AB strain, were maintained on a 14-h light/10-h dark cycle to stimulate spawning (Westerfield, 1994), and embryos were maintained in Holtfreter's solution at $28.5^{\circ} \mathrm{C}$ and staged according to Kimmel et al. (1995). Embryos used in whole-mount in situ hybridization were raised in $0.003 \%$ PTU (Sigma) to prevent pigmentation.

\section{Molecular cloning and sequence analysis}

Total RNA was purified with Trizol reagent (Invitrogen) from 36-h postfertilization (hpf) embryos, and cDNA was synthesized using random primers and reverse transcriptase. 5'-Rapid amplification of cDNA ends (5'-RACE) was carried out using the DD SMART RACE cDNA Amplification Kit according to the manufac- 
turer protocol (Clontech). The 5'-RACE fragment of zRBM22 was amplified with the following primer: 5'-ATGGCGACGCCTTTGGGACT-3'. The cDNA fragment was cloned into the pGEM-T Easy vector (Invitrogen).

The nucleotide sequence and predicted amino acid sequence homologies were analyzed via the GenomeNet WWW server using BLASTN and BLASTP searches of the non-redundant database. Phylogenetic analysis was performed using the CLUSTAL W program.

\section{Reverse transcription-polymerase chain reaction (RT-PCR) detection of ZRBM22 mRNA}

Total RNA from zebrafish embryos at different stages were isolated with the Trizol reagent. cDNA was obtained with the One-Step RT-PCR kit (Takara). $\beta$-actin was used as internal control for normalization. $\beta$-actin and zRBM22 primers were designed with DNAStar package. $\beta$-actin was amplified with the forward primer 5'-ATGGATGATGAAATTGCCGCAC-3' and reverse primer 5'-ACCATCACCAGAGTCCATCACG-3'. zRBM22 primers were forward: 5'-GCGCGCACCACACCGTACTA-3' and reverse: 5'-GGTGGAGGCTCGCATCAGCA-3'.

\section{In situ hybridization}

The cDNA fragment of zRBM22 was used to make RNA probes. T3 or T7 RNA polymerase was utilized for the synthesis of either sense or antisense digoxigenin (DIG)labeled ribonucleotide probes (Ambion, USA).

Whole-mount in situ hybridization of zebrafish embryos was performed as previously described (Schulte-Merker et al., 1992). Briefly, embryos at different stages were fixed in $4 \%$ paraformaldehyde overnight at $4{ }^{\circ} \mathrm{C}$, followed by washing twice with PBS. Embryos were then dehydrated in $100 \%$ methanol overnight. Samples were rehydrated followed by a brief incubation with $10 \mathrm{mg} / \mathrm{mL}$ proteinase $\mathrm{K}$ (incubation time depended on the stage of the embryos) to increase tissue permeability, and the embryos were refixed with 4\% paraformaldehyde in PBS for $20 \mathrm{~min}$. After washing with PBS, hybridization was carried out in $500 \mathrm{~mL}$ hybridization buffer $(50 \%$ formamide, $5 X$ SSC, $500 \mathrm{mg} / \mathrm{mL}$ yeast transfer RNA, $0.1 \%$ Tween $20,1 \%$ CHAPS and $100 \mathrm{mg} / \mathrm{mL}$ heparin) containing 40 ng DIG-labeled RNA probes, at $55^{\circ} \mathrm{C}$ overnight. After extensive washing, anti-DIG antibodies conjugated with alkaline phosphatase were used to localize mRNA. Color development was carried out using BM-purple under conditions recommended by the manufacturer (Boehringer Mannheim, USA).

\section{Microinjection of antisense morpholino oligonucleotides}

Morpholino oligonucleotides were obtained from Gene Tools (LLC, USA). A targeted gene knockdown approach was utilized as reported by Nasevicius and Ekker (2000). The sequence of zRBM22 antisense morpholino was 5'-GTTGGATCCCAAAGACGTCGCCATT-3'. Stock solutions of morpholino oligonucleotides were prepared at $3.0 \mathrm{mM}$ and the procedure for the injection of diluted concentrations followed that described by Nasevicius and Ekker (2000). Individual embryos 
were injected at the 1-cell stage using volumes established by Nasevicius and Ekker using a PLI-90 injector system (Harvard Apparatus).

Control oligonucleotides included scrambled and 4-base mismatch morpholino oligonucleotides. Following injection of various amounts of oligonucleotides, embryos of antisense and control groups were monitored for 15 and $24 \mathrm{hpf}$, and the number of embryos with an abnormal versus normal phenotype was determined. Images were obtained using a Nikon dissecting microscope.

\section{RESULTS AND DISCUSSION}

\section{Cloning and structural analysis of zRBM22}

To obtain zebrafish RBM22 cDNA clones, a human RBM22 cDNA clone corresponding to its open reading frame was used to screen a zebrafish oligo dT-primed cDNA library. One full-length zebrafish RBM22 (zRBM22) cDNA clone was obtained from the screening. Analysis of the deduced amino acid sequence of zRBM22 indicated that this open reading frame encodes a protein of 425 amino acids, which contains a conserved RNAbinding domain, a zinc finger of the unusual type C-x8-C-x5-C-x3-H. The C-terminus is unusually rich in the amino acids Gly and Pro, as shown in Figure 1 (obtained from: http:// www.uniprot.org/uniprot/Q6NZZ9).

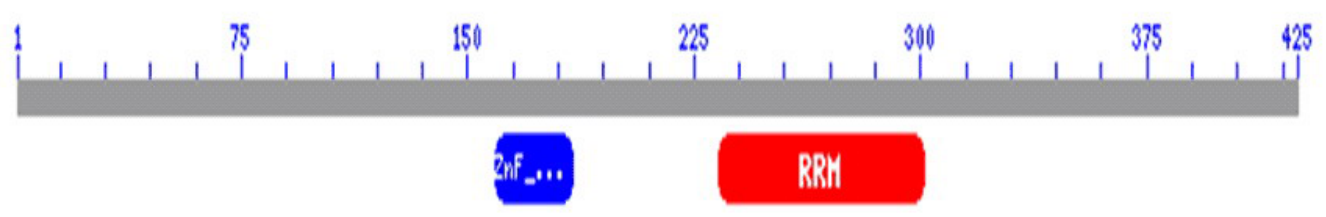

\section{Zn-finger \\ RNA-binding region RNP-1 (RNA recognition motif)}

Figure 1. A schematic illustration of zRBM22. Amino acid sequence was deduced from the zRBM22 cDNA. zRBM22 contains a conserved RNA-binding domain and a zinc finger of the unusual type C-x8-C-x5-C-x3-H.

Figure 2A shows the aligned protein sequences of RBM22 of human, monkey, mouse, rat, bovine, frog, and zebrafish. The polygenetic tree displays that zebrafish and frog share over $90 \%$ similarity to RBM22 at the amino acid level with other species. Human, monkey, mouse, rat, and bovine even have 100\% similarity to RBM22 (Figure 2B). It is well known that the conservation of a gene in evolution means it has an important biological function. According to evolutionary opinion, the more important a gene is, the more selection pressure it undergoes, and then, the more conserved the gene is. Therefore, the high conversation of RBM22 indicates that it could have an important biological function. 
A

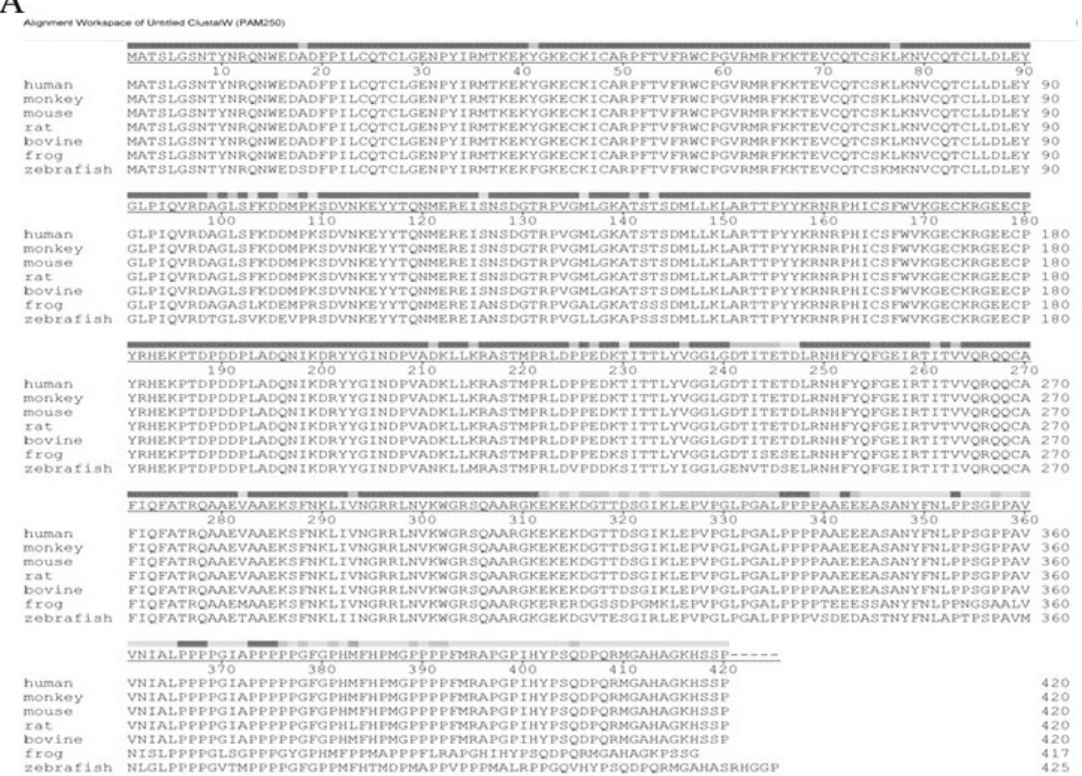

B

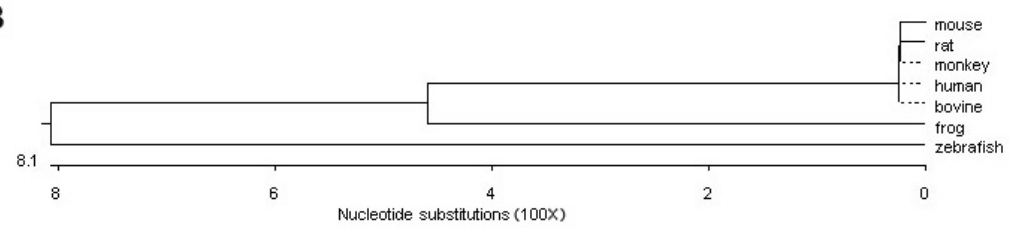

Figure 2. Alignment (A) and phylogenetic tree (B) of the amino acid sequences of RBM22 from various organisms. The amino acid sequences of human, monkey, mouse, rat, bovine, frog, and zebrafish RBM22 were obtained from the GenBank database with accession numbers: Q9NW64, Q4R4J1, Q8BHS3, Q4V7D7, Q3B7L8, Q7ZXB5, and Q6NZZ9, respectively.

\section{Expression pattern of zRBM22 during embryogenesis}

To determine the spatial and temporal expression pattern of zRBM22, whole-mount in situ analysis was performed. As shown in Figure 3, in situ signal for zRBM22 was detected as early as the 1-cell stage (Figure 3A) and continued through the 8-cell stage (Figure 3B), 32-cell stage (Figure 3C), 15-hpf stage (Figure 3D), and 24-hpf stage (Figure 3E). RT-PCR analysis confirmed the presence of zRBM22 mRNA as reflected in the in situ studies (Figure $3 \mathrm{~F}$ ) and the amount of zRBM22 mRNA at different stages being nearly equal. The data of RTPCR and in situ analysis at the 1-cell stage showed that RBM22 was a maternal origin gene. Furthermore, RBM22 expressed without any spatial and temporal specificity; that is, it can be detected in different developmental stages and parts of the fish. These results indicated that RBM22 could play an essential role in zebrafish development. 

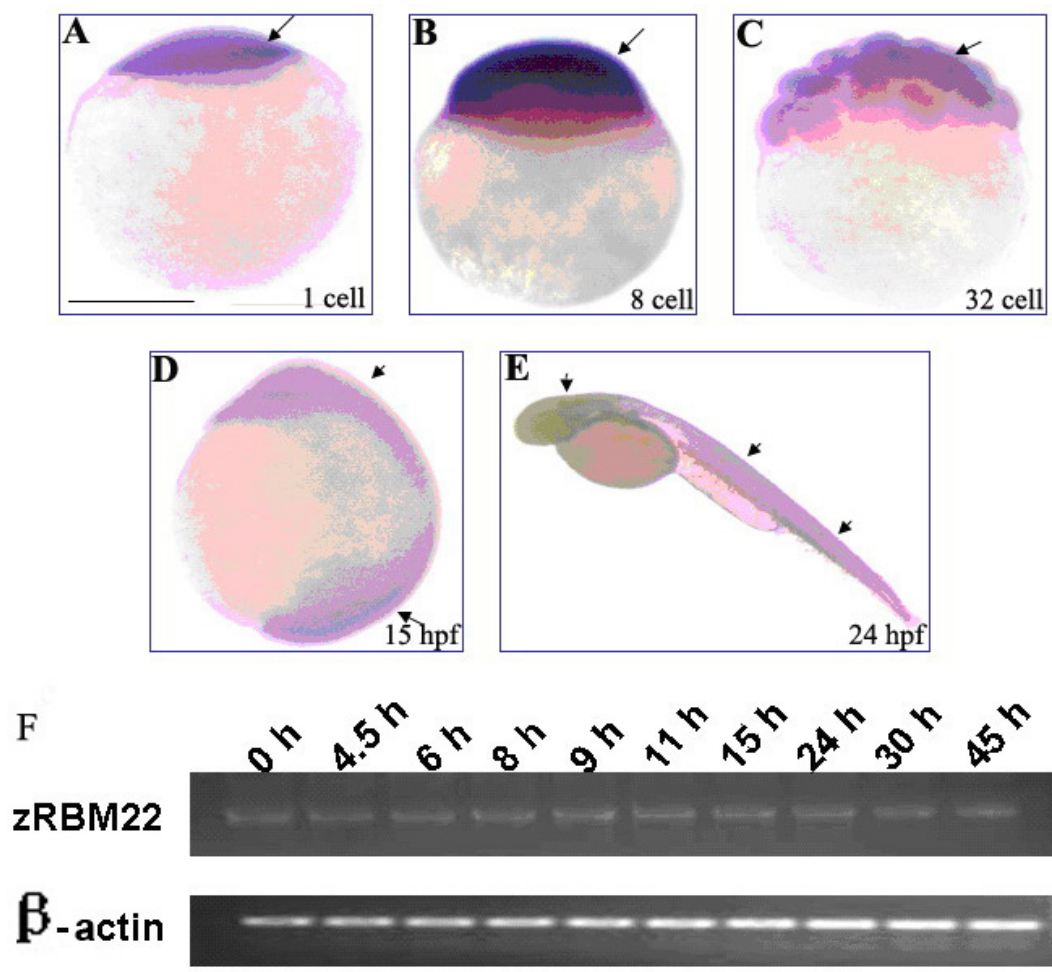

Figure 3. In situ analysis and RT-PCR analysis of zRBM22 during zebrafish embryogenesis. zRBM22 mRNA was detected during early stages of zebrafish development. An in situ signal was detected as early as the 1-cell stage (A; arrow) and through the 8-cell stage (B; arrow), the 32-cell stage (C; arrow), and the 15-hpf stage (D; arrows). A signal along the entire anterior to posterior axis (arrows) was seen in embryos at $24 \mathrm{hpf}$ (E). RT-PCR analysis of zRBM22 is shown in $F$. mRNA expression levels for zRBM22 and $\beta$-actin (normalizing standard) were determined at 10 stages of development: 1-cell stage, 4.5-h, 6-h, 8-h, 9-h, 11-h, 15-h, 24-h, 30-h, and 45-h stage. hpf = hours post-fertilization. Scale bar equals $250 \mu \mathrm{m}$ for A-D and $100 \mu \mathrm{m}$ for E.

\section{Loss-of-function analysis of zRBM22}

To determine if RBM22 expression is essential for zebrafish development, we performed a loss-of-function analysis using the morpholino oligonucleotide antisense morpholino oligonucleotide, which prevents the translation of zRBM22 mRNA. In the present study, morpholino oligonucleotides were injected into embryos at the 1- to 4-cell stage. As shown in Figure 4, injected morpholino oligonucleotides resulted in an abnormal phenotype with perturbation along the entire anterior-posterior axis and arrested development of the head and tail as viewed from $15 \mathrm{hpf}$ (Figure 4B) through $24 \mathrm{hpf}$ (Figure 4D) and $32 \mathrm{hpf}$ (Figure 4F). However, the mismatch controls had no effect on normal development (Figure 4A, C and E). The results reveal that RBM22 is an indispensable gene in zebrafish embryogenesis. 

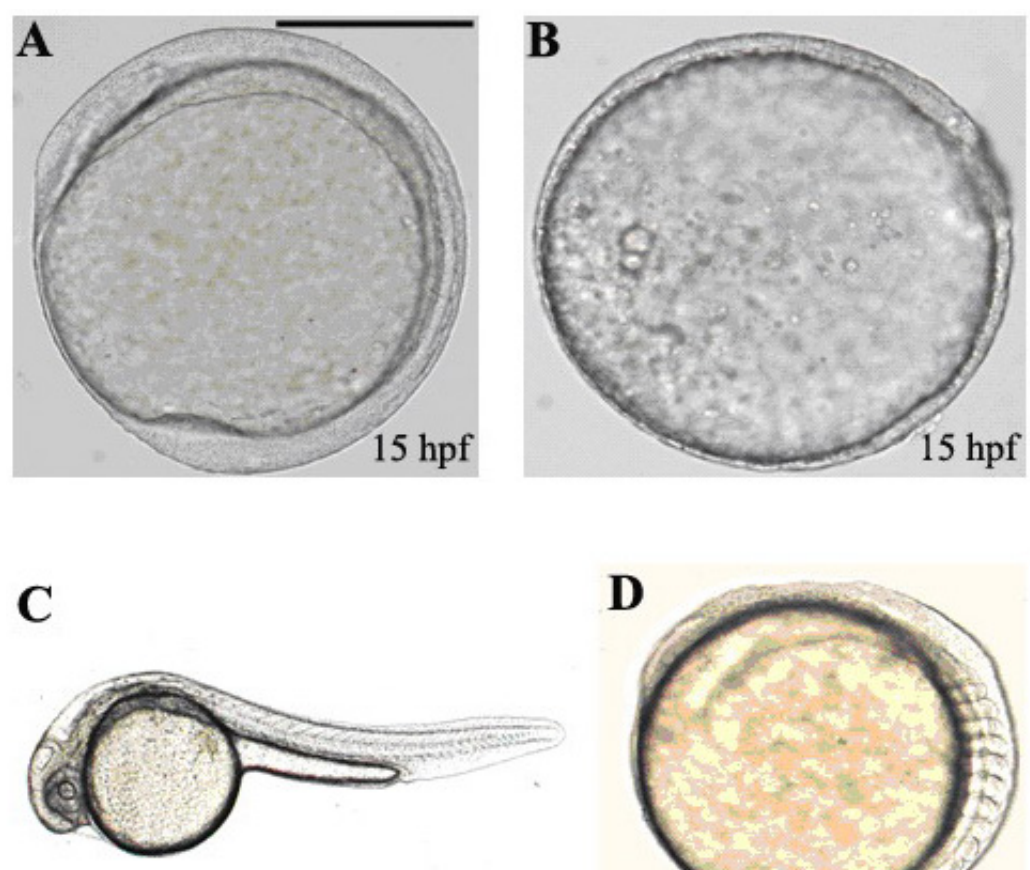

$24 \mathrm{hpf}$
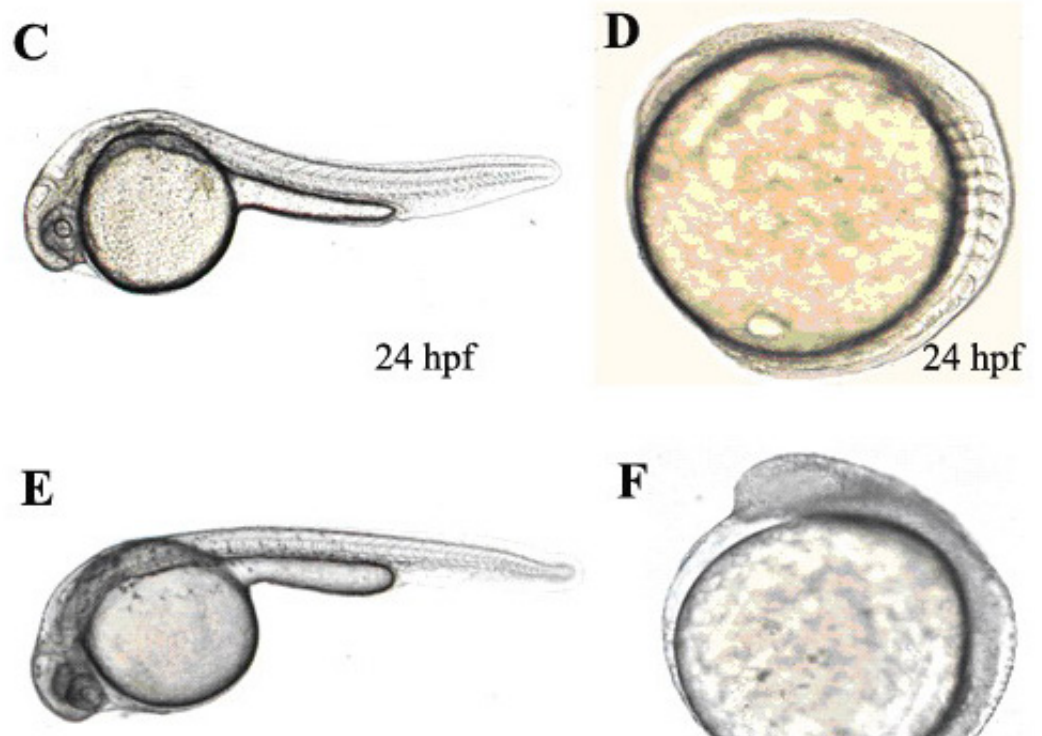

$32 \mathrm{hpf}$

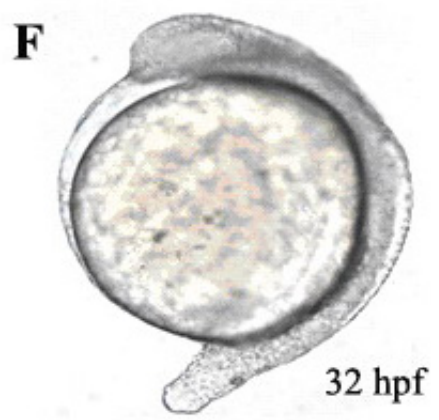

Figure 4. Effects of antisense morpholino oligonucleotides against zRBM22 on zebrafish development. Images of control embryos are shown for the 15-hpf (A), 24-hpf (C) and 32-hpf (E) stages. Embryos with a developmental blocking phenotype following injection of morpholino oligonucleotides to zRBM22 into 1- to 4-cell stage embryos are shown in $B(15 \mathrm{hpf}), D(24 \mathrm{hpf})$ and $F(32 \mathrm{hpf})$. hpf= hours post-fertilization. Scale bar equals $250 \mu \mathrm{m}$.

Montaville and co-workers (2006) have reported that increased translation of zRBM22 using an mRNA microinjection approach cannot affect zebrafish early development. Proteomic analysis indicated that as one of the core proteins of a purified human spliceosomal C complex, RBM22 is able to carry out the second step of splicing (Xu and Friesen, 2001; 
Bessonov et al., 2008; Barboric et al., 2009). According to over-expression, proteomic analysis and our morpholino oligonucleotide result, we propose that the main function of RBM22 is exclusively to serve as a structural component of the splicesome. As a structural protein, the overdose RBM22p cannot affect normal cell physiological functions. However, insufficient RBM22p affects the common assembly of the pre-splicesome and the maturation of pre-mRNA. The consequence in embryogenesis is a block of development

Taken together, zRBM22 is expressed during the earliest stages of zebrafish development, and functional studies indicate that zRBM 22 has an important role in the embryogenesis of this vertebrate system.

\section{ACKNOWLEDGMENTS}

The authors thank Dr. Fanxin Ma for his help. Research supported by the National Basic Research Program of China (grant \#2004CB51880).

\section{REFERENCES}

Barboric M, Lenasi T, Chen H, Johansen EB, et al. (2009). 7SK snRNP/P-TEFb couples transcription elongation with alternative splicing and is essential for vertebrate development. Proc. Natl. Acad. Sci. U. S. A. 106: 7798-7803.

Bessonov S, Anokhina M, Will CL, Urlaub H, et al. (2008). Isolation of an active step I spliceosome and composition of its RNP core. Nature 452: 846-850.

Chen YI, Moore RE, Ge HY, Young MK, et al. (2007). Proteomic analysis of in vivo-assembled pre-mRNA splicing complexes expands the catalog of participating factors. Nucleic Acids Res. 35: 3928-3944.

Jurica MS and Moore MJ (2003). Pre-mRNA splicing: awash in a sea of proteins. Mol. Cell 12: 5-14.

Kimmel CB, Ballard WW, Kimmel SR, Ullmann B, et al. (1995). Stages of embryonic development of the zebrafish. Dev. Dyn. 203: 253-310.

Kramer A (1996). The structure and function of proteins involved in mammalian pre-mRNA splicing. Annu. Rev. Biochem. 65: 367-409.

Montaville P, Dai Y, Cheung CY, Giller K, et al. (2006). Nuclear translocation of the calcium-binding protein ALG-2 induced by the RNA-binding protein RBM22. Biochim. Biophys. Acta 1763: 1335-1343.

Nasevicius A and Ekker SC (2000). Effective targeted gene 'knockdown' in zebrafish. Nat. Genet. 26: 216-220.

Nilsen TW (2000). The case for an RNA enzyme. Nature 408: 782-783.

Schulte-Merker S, Ho RK, Herrmann BG and Nusslein-Volhard C (1992). The protein product of the zebrafish homologue of the mouse $\mathrm{T}$ gene is expressed in nuclei of the germ ring and the notochord of the early embryo. Development 116: 1021-1032.

Westerfield M (1994). The Zebrafish Book: A Guide for the Laboratory Use of Zebrafish (Brachidanio rerio). University of Oregon Press, Eugene.

Will CL and Luhrmann R (2001). Spliceosomal UsnRNP biogenesis, structure and function. Curr. Opin. Cell Biol. 13: 290-301.

Xu D and Friesen JD (2001). Splicing factor slt1 1p and its involvement in formation of U2/U6 helix II in activation of the yeast spliceosome. Mol. Cell Biol. 21: 1011-1023.

Xu D, Field DJ, Tang SJ, Moris A, et al. (1998). Synthetic lethality of yeast slt mutations with U2 small nuclear RNA mutations suggests functional interactions between U2 and U5 snRNPs that are important for both steps of premRNA splicing. Mol. Cell Biol. 18: 2055-2066. 